\title{
High prevalence of low dairy calcium intake and association with insomnia, anxiety, depression and musculoskeletal pain in university students from Jordan
}

\author{
Mohammad J Alkhatatbeh 1,* , Hala N Khwaileh² and Khalid K Abdul-Razzak' \\ 'Department of Clinical Pharmacy, Faculty of Pharmacy, Jordan University of Science and Technology, Irbid 22110 , \\ Jordan: ${ }^{2}$ Department of Nutrition and Food Technology, Faculty of Agriculture, Jordan University of Science and \\ Technology, Irbid, Jordan
}

Submitted 9 March 2020: Final revision received 14 July 2020: Accepted 20 July 2020: First published online 24 August 2020

\begin{abstract}
Objective: To assess dairy Ca intake and investigate its relationship with insomnia and other common co-morbidities including anxiety, depression and musculoskeletal pain (MSP) among university students.

Design: Cross-sectional study.

Setting: University, Irbid, Jordan.

Participants: Male and female individuals ( $n$ 1000), aged $20.87 \pm 2.69$ years.

Results: Low dairy Ca intake $(<1000 \mathrm{mg} / \mathrm{d})$ was reported by $96.5 \%$ of participants, and moderate to severe insomnia reported by $15.6 \%$ of participants. Abnormal anxiety and depression scores were reported by 26.2 and $18.0 \%$ of participants, respectively. MSP was reported by $42.9 \%$ of participants. Participants with moderate to severe insomnia had lower dairy $\mathrm{Ca}$, higher anxiety and depression scores and higher measures of MSP compared to participants with no insomnia $(P$-values $<0.05)$. Dairy Ca was weakly inversely correlated with Insomnia Severity Index (ISI) score, depression score and measures of MSP ( $P$-values < 0.05). Regression analysis indicated that insomnia was predicted by low dairy $\mathrm{Ca}$, anxiety, depression, MSP and smoking $(P$-values $<0.05)$. Both anxiety and depression were predicted by increased ISI score $(P$-values $<0.05)$, while depression alone was predicted by low dairy Ca $(P$-value $<0.01)$. MSP was predicted by increased ISI and anxiety scores $(P$-values $<0.05)$.

Conclusions: Low dairy Ca was highly prevalent and associated with insomnia and depression among university students. Individuals should be advised to increase dietary $\mathrm{Ca}$ intake to achieve the recommended daily amount. Further research is required to investigate a potential causal relationship between low $\mathrm{Ca}$ and both insomnia and its related co-morbidities.
\end{abstract}

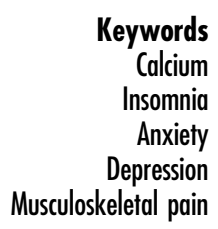

eywords

Anxiety

Depression

Musculoskeletal pain
Insomnia is a type of sleep disorder that is characterised by difficulty in sleep initiation or maintenance at night and is usually accompanied by daytime symptoms such as fatigue, sleepiness, irritability and loss of concentration $^{(1)}$. Transient insomnia occurs at a rate of approximately $30 \%$ among adults, ${ }^{(2)}$ while chronic insomnia is reported in approximately $\sim 10-30 \%$ of adults and more common in those who are divorced or widowed, elderly, female or suffer from psychiatric or medical diseases ${ }^{(2,3)}$. Chronic insomnia may adversely affect daily functioning and if prolonged may be associated with increased risk of deleterious health conditions including obesity, diabetes mellitus, hypertension and other $\mathrm{CVD}^{(4,5)}$.
There is evidence to suggest that increased dietary Ca intake, which is mainly obtained from dairy products ${ }^{(6)}$, is associated with improved quality of sleep and reduced insomnia $^{(7,8)}$. Total plasma Ca was found to be directly related to sleep as a diurnal variation in its concentration was detected during normal sleep ${ }^{(9)}$. In addition, brain $\mathrm{Ca}$ is proposed to have a role in regulating the duration of sleep in mammals ${ }^{(10)}$. The effect of dairy products on sleep could also be due to their content of tryptophan, ${ }^{(11)}$ as $\mathrm{Ca}$ is involved in producing melatonin, the sleep-inducing neurosecretory hormone, from tryptophan in the brain ${ }^{(8)}$.

Because $\mathrm{Ca}$ is necessary for proper musculoskeletal function $^{(12)}$, low plasma Ca levels are usually associated 
with paresthesia in extremities, fatigue and muscle cramps and spasms that can be very painful ${ }^{(13)}$. These symptoms may lead to some psychiatric conditions including anxiety and depression, which can also be present due to hypocalcaemia itself ${ }^{(13)}$. Our previous work showed independent associations between non-cardiac musculoskeletal pain (MSP) and anxiety, as well as between anxiety and low dietary Ca intake, suggesting that anxiety and MSP could be associated with low plasma Ca levels ${ }^{(14)}$. MSP may also be associated with insomnia and the two conditions could co-occur with each of them increasing the risk of the other ${ }^{(15)}$. In addition, several studies have reported a high co-morbidity between insomnia, anxiety and depression ${ }^{(16-19)}$.

The aim of the current study was to assess dairy $\mathrm{Ca}$ intake and its relationship to insomnia and other common co-morbidities including anxiety, depression and MSP in Jordanian university students whose academic achievement is often negatively impacted by these conditions. In Jordan, dietary $\mathrm{Ca}$ is mainly obtained from dairy products that are available in the Jordanian market including cheese, yogurt, milk and labanah. These types of food are very popular and rich in $\mathrm{Ca}^{(14,20)}$. We hypothesised that low dairy $\mathrm{Ca}$ intake is highly prevalent in this population and is associated with insomnia, anxiety, depression and MSP. Understanding the relationship between Ca intake and these common ailments may be effective in improving insomnia and psychological symptoms in the study population.

\section{Materials and methods}

\section{Study design and participants}

A single institution questionnaire-based cross-sectional study was conducted between September 2019 and January 2020 at Jordan University of Science and Technology, Irbid, Jordan. The study sample consisted of 1000 enrolled undergraduate students recruited from all schools of the university. Participants were divided into two groups: 'medical students' who were specialising in medicine, dentistry, pharmacy, nursing or applied medical sciences and 'non-medical students' who were specialising in engineering, computer sciences, science and art, agriculture or architecture and design. The study procedure was ethically approved by the Institutional Review Board of the university. Written informed consent was obtained from all participants before they personally completed the questionnaires. A research assistant was available to clarify any inquiries from the participants.

\section{Data collection}

Self-reported data collection included: age, height, weight, marital status, college, academic level, smoking, number of family members, family income, exercise, as well as history of medical illness (including psychological illnesses) and
Ca supplementation during the previous month. BMI was calculated from weight and height in $\mathrm{kg} / \mathrm{m}^{2}$.

\section{Assessment of daily dairy calcium intake}

Participants were asked to report the daily frequency and type of dairy product consumed including cheese, yogurt, milk and labanah as previously described ${ }^{(14,20)}$. These are the most common dairy products available in the Jordanian market and they represent the major sources of dietary $\mathrm{Ca}$ in this population ${ }^{(14,20)}$. Frequency of dairy intake was defined as $0,1,2,3$ or more servings/d, ${ }^{(14,20)}$ where a single serve was defined as: 1 ounce of cream cheese (20 mg Ca), 1 ounce of cheddar cheese (162 mg Ca), one cup $(240 \mathrm{ml})$ of milk or yogurt (300 $\mathrm{mg} \mathrm{Ca})$ and two tablespoons $(2 \mathrm{Oz})$ of labanah $(100 \mathrm{mg} \mathrm{Ca})^{(14,20)}$. Daily Ca intake was then calculated in $\mathrm{mg} / \mathrm{d}$. None of the participants reported intake of Ca supplements.

\section{Assessment of insomnia and its severity}

Insomnia was assessed using an Arabic version of the wellvalidated Insomnia Severity Index (ISI) ${ }^{(21)}$ that was previously translated from English to Arabic using the standard forward-backward translation method ${ }^{(22)}$. The Arabic translation was also pilot tested on thirty undergraduate university students who were not participants in the current study. The ISI is a self-administered seven question survey that measures severity of insomnia experienced over the previous 2 weeks. The first three questions assess the difficulty in falling asleep, difficulty in staying asleep and problems with waking up prematurely. The remaining four questions assess how the subject's satisfaction with their current sleep pattern, how noticeable it is to others that there may be sleep problem impairing the subject's quality life, whether the subject is worried about their current sleep pattern and how much the current sleep pattern is interfering with the subject's daily functioning. Each of these questions is answered by selecting a number from 0 to 4 that describes the severity of the problem and the ISI score (0-28) is calculated by adding the scores of all questions together. Participants with ISI scores from 0 to 7 are considered to have no clinically significant insomnia, participants with ISI scores from 8 to 14 are classified as having subthreshold insomnia and participants with ISI scores from 15 to 28 are considered to have clinical insomnia (moderate to severe).

\section{Assessment of psychological symptoms (anxiety and depression)}

Anxiety and depression symptoms were assessed using an Arabic version of the well-validated Hospital Anxiety and Depression Scale (HADS) ${ }^{(23)}$. The standard forwardbackward translation method was also used to translate the HADS from English to Arabic as previously described ${ }^{(14)}$. The Arabic translation was also pilot tested on thirty undergraduate university students who were not participants in the current study. The HADS consists of seven questions that 
assess anxiety and seven questions that assess depression. Each question is answered by selecting a number from 0 to 3 considering to severity. Thus, the total score for both anxiety and depression ranges from 0 to 21. Participants were considered normal (HADS-anxiety or depression scores from 0 to 7 ), borderline (HADS-anxiety or depression scores from 8 to 10) or abnormal (HADS-anxiety or depression scores from 11 to 21$)^{(23)}$

\section{Assessment of musculoskeletal pain}

As previously described ${ }^{(24)}$, participants were asked to report the sites and severity of chronic MSP. Areas of MSP were indicated using an illustration of the human body consisting of twelve sites including neck, shoulders, lower back, hands, wrists, palms, arms, upper legs, knees, lower legs, hips and feet. The number of painful body sites was summed. Additionally, participants were asked to report the overall intensity of MSP during the last month using a 0-10 numerical rating scale (0 reflects no MSP and 10 reflects the maximum intensity of MSP) ${ }^{(24)}$.

\section{Statistical analysis}

Analysis was performed using the IBM SPSS Statistics software version 23. Data were presented as frequency (\%), mean \pm SD or median (25th-75th percentiles) as appropriate. Differences in variables according to insomnia status were determined using one-way ANOVA with Tukey posthoc test for multiple comparisons, Kruskal-Wallis one-way ANOVA or $\chi^{2}$ test as appropriate. Correlation analysis between ISI score and other continuous variables was performed using either Pearson's or Spearman's correlation test as appropriate. Predictors of insomnia, anxiety and depression were determined using multiple linear regression analysis. Predictors of MSP were determined using binary logistic regression analysis. All $P$-values were two-tailed and considered statistically significant at $<0.05$.

\section{Results}

\section{General characteristics of the participants}

One thousand participants $(36.7 \%$ males and $63.3 \%$ females) were recruited, consisting of $52.4 \%$ medical and $47.6 \%$ non-medical university students, whose mean age was $20.87 \pm 2.69$ years. According to their academic level, $55.3 \%$ of participants were in their 1 st or 2 nd year (junior students) and $44.7 \%$ of participants were in their 3rd-6th year (senior students). Participants had a mean BMI of $23.75 \pm 4.56 \mathrm{~kg} / \mathrm{m}^{2}$ and only $14.7 \%$ of participants were current smokers. Other characteristics of participants are reported in Table 1.

\section{Daily dairy calcium intake}

Most participants (96.5\%) reported a daily dairy Ca intake that was well below the recommended daily intake of
$1000 \mathrm{mg} / \mathrm{d}$ for adults, ${ }^{(25)}$ with an overall median (25th75th percentiles) of $288.12(130 \cdot 95-487.72) \mathrm{mg} / \mathrm{d}$.

\section{Prevalence of insomnia and its severity}

According to the ISI scoring system, $15.6 \%$ of participants suffered from moderate to severe clinical insomnia, $45.0 \%$ of participants had subthreshold insomnia and $39.4 \%$ of participants had no clinically significant insomnia. The mean ISI score for all participants was 9.31 $\pm 5 \cdot 46$.

\section{Prevalence of psychological symptoms (anxiety and depression)}

According to the HADS scoring system, abnormal HADSanxiety scores were reported in $26.2 \%$ of participants and abnormal HADS-depression scores were reported in $18.0 \%$ of participants. Additionally, $22.5 \%$ of participants recorded borderline HADS-anxiety scores and 24.3\% of participants had borderline HADS-depression scores. The remaining participants had normal HADS-anxiety (51.3\%) and depression (57.7\%) scores. The mean HADSanxiety and depression scores for all participants were $7 \cdot 8 \pm 4 \cdot 33$ and $7 \cdot 13 \pm 3 \cdot 7$, respectively.

\section{Prevalence of musculoskeletal pain}

MSP was reported by $42.9 \%$ of participants. The median (25th-75th percentiles) MSP intensity (out of 10) during the previous month was $0(0-4)$ and the median (25th75th percentiles) number of painful body sites (out of 12) was $0(0-1 \cdot 75)$.

\section{Differences between study variables according to insomnia status}

As shown in Table 1, moderate to severe insomnia was more prevalent in non-medical students compared with medical students ( $P$-value $=0 \cdot 04)$. Current smokers, participants with a history of medical illness and those who reported MSP during the previous month reported higher frequencies of moderate to severe insomnia compared with subthreshold insomnia and no clinically significant insomnia ( $P$-values $=0.01,<0.01,<0.001$, respectively $)$. Participants with moderate to severe insomnia had higher HADS-anxiety and depression scores, higher MSP intensity during the previous month, more painful body sites and lower daily dairy Ca intake compared to participants with subthreshold insomnia and participants with no clinically significant insomnia ( $P$-values $<0 \cdot 001)$.

\section{Correlation between Insomnia Severity Index score, Hospital Anxiety and Depression Scale-anxiety and depression scores, musculoskeletal pain and daily dairy calcium intake}

As shown in Table 2, there was a relatively strong correlation between the ISI and HADS-anxiety scores $(r=0.43$, $P$-value $<0.001)$, and moderate correlations between ISI 


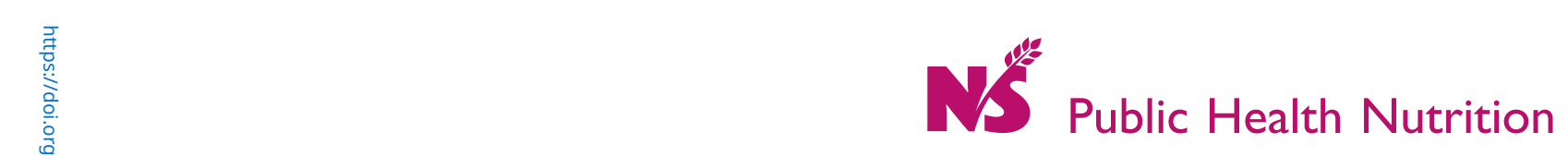

\begin{tabular}{|c|c|c|c|c|c|c|c|c|c|}
\hline & \multicolumn{2}{|c|}{ Total $(n 1000)$} & \multicolumn{2}{|c|}{$\begin{array}{c}\text { No clinically significant } \\
\text { insomnia (ISI score = } \\
0-7)(n \text { 394) }\end{array}$} & \multicolumn{2}{|c|}{$\begin{array}{l}\text { Subthreshold insomnia } \\
\text { (ISI score = 8-14) (n 450) }\end{array}$} & \multicolumn{2}{|c|}{$\begin{array}{c}\text { Moderate to severe } \\
\text { clinical insomnia (ISI } \\
\text { score = 15-28) }(n \text { 156) }\end{array}$} & \multirow[b]{2}{*}{$P^{*}$} \\
\hline & $n$ & $\%$ & $n$ & $\%$ & $n$ & $\%$ & $n$ & $\%$ & \\
\hline \multicolumn{10}{|l|}{ Age } \\
\hline $\begin{array}{l}\text { Mean } \\
\text { SD }\end{array}$ & $\begin{array}{r}20.87 \\
2.69\end{array}$ & & $\begin{array}{r}20.95 \\
2.72\end{array}$ & & $\begin{array}{r}20.83 \\
2.37\end{array}$ & & $\begin{array}{r}21.01 \\
2.60\end{array}$ & & 0.68 \\
\hline \multicolumn{10}{|l|}{ Gender } \\
\hline Male & 367 & $36 \cdot 7$ & 140 & 35.5 & 165 & 36.7 & 62 & 39.7 & 0.65 \\
\hline Female & 633 & 63.3 & 254 & 64.5 & 285 & 63.3 & 94 & $60 \cdot 3$ & \\
\hline \multicolumn{10}{|l|}{ Marital status } \\
\hline Single & 978 & 97.8 & 381 & $96 \cdot 7$ & 445 & 98.9 & 152 & 97.4 & 0.08 \\
\hline Married & 22 & $2 \cdot 2$ & 13 & 3.3 & 5 & $1 \cdot 1$ & 4 & $2 \cdot 6$ & \\
\hline \multicolumn{10}{|l|}{ College } \\
\hline Medical & 524 & 52.4 & 214 & $54 \cdot 3$ & 243 & $54 \cdot 0$ & 67 & $42 \cdot 9$ & 0.04 \\
\hline Non-medical & 476 & $47 \cdot 6$ & 180 & $45 \cdot 7$ & 207 & $46 \cdot 0$ & 89 & $57 \cdot 1$ & \\
\hline \multicolumn{10}{|l|}{ Academic level } \\
\hline Junior students (1st and 2nd years) & 553 & $55 \cdot 3$ & 214 & $54 \cdot 3$ & 253 & $56 \cdot 2$ & 86 & $55 \cdot 1$ & 0.87 \\
\hline Senior students (3rd-6th year) & 447 & 44.7 & 180 & $45 \cdot 7$ & 197 & 43.8 & 70 & 44.9 & \\
\hline \multicolumn{10}{|l|}{ BMI $\left(\mathrm{kg} / \mathrm{m}^{2}\right)$} \\
\hline Mean & 23.75 & & 23.92 & & 23.64 & & 23.63 & & 0.73 \\
\hline \multirow{2}{*}{\multicolumn{10}{|c|}{ Smoking }} \\
\hline & & & & & & & & & \\
\hline Yes & 147 & $14 \cdot 7$ & 43 & $10 \cdot 9$ & 72 & $16 \cdot 0$ & 32 & 20.5 & 0.01 \\
\hline No & 853 & 85.3 & 351 & $89 \cdot 1$ & 378 & 84.0 & 124 & 79.5 & \\
\hline \multicolumn{10}{|l|}{ Number of family members } \\
\hline Mean & 6.47 & & $6 \cdot 33$ & & $6 \cdot 56$ & & $6 \cdot 57$ & & 0.26 \\
\hline SD & 1.74 & & 1.74 & & 1.68 & & 1.89 & & \\
\hline \multicolumn{10}{|l|}{ Average family income } \\
\hline$\leq 500 \mathrm{JD}$ & 246 & $24 \cdot 6$ & 98 & 24.9 & 103 & 22.9 & 45 & $28 \cdot 8$ & \\
\hline $501-1000$ JD & 402 & $40 \cdot 2$ & 162 & $41 \cdot 1$ & 175 & 38.9 & 65 & 41.7 & 0.31 \\
\hline >1000 JD & 352 & $35 \cdot 2$ & 134 & $34 \cdot 0$ & 172 & 38.2 & 46 & 29.5 & \\
\hline \multicolumn{10}{|l|}{ Regular exercise } \\
\hline Yes & 664 & $66 \cdot 4$ & 273 & $69 \cdot 3$ & 294 & $65 \cdot 3$ & 97 & $62 \cdot 2$ & 0.23 \\
\hline No & 336 & 33.6 & 121 & $30 \cdot 7$ & 156 & 34.7 & 59 & $37 \cdot 8$ & \\
\hline \multicolumn{10}{|c|}{ History of medical illness during previous month } \\
\hline Yes & 106 & $10 \cdot 6$ & 35 & 8.9 & 42 & $9 \cdot 3$ & 29 & $18 \cdot 6$ & $<0.01$ \\
\hline No & 894 & 89.4 & 359 & $91 \cdot 1$ & 408 & 90.7 & 127 & 81.4 & \\
\hline \multicolumn{10}{|l|}{ HADS-anxiety score $(0-21)$} \\
\hline Mean & $7 \cdot 8$ & & $5 \cdot 99 \dagger \ddagger$ & & $8 \cdot 22+\S$ & & $11 \cdot 18 \ddagger \S$ & & 0.000 \\
\hline SD & 4.33 & & 3.91 & & 3.88 & & $4 \cdot 30$ & & \\
\hline Normal (0-7) & 513 & 51.3 & 274 & 69.5 & 206 & $45 \cdot 8$ & 33 & $21 \cdot 2$ & \\
\hline Borderline (8-10) & 225 & 22.5 & 64 & $16 \cdot 2$ & 125 & $27 \cdot 8$ & 36 & 23.1 & 0.000 \\
\hline Abnormal (11-21) & 262 & $26 \cdot 2$ & 56 & $14 \cdot 2$ & 119 & $26 \cdot 4$ & 87 & $55 \cdot 8$ & \\
\hline \multicolumn{10}{|l|}{ HADS-depression score (0-21) } \\
\hline Mean & $7 \cdot 13$ & & $6 \cdot 09 \dagger \ddagger$ & & $7 \cdot 27^{\dagger \S}$ & & $9 \cdot 37^{\ddagger \S}$ & & 0.000 \\
\hline SD & 3.7 & & 3.36 & & 3.48 & & 4.08 & & \\
\hline
\end{tabular}




\begin{tabular}{|c|c|c|c|c|c|c|c|c|c|}
\hline & \multicolumn{2}{|c|}{ Total $(n 1000)$} & \multicolumn{2}{|c|}{$\begin{array}{l}\text { No clinically significant } \\
\text { insomnia (ISI score = } \\
0-7)(n 394)\end{array}$} & \multicolumn{2}{|c|}{$\begin{array}{l}\text { Subthreshold insomnia } \\
\text { (ISI score = 8-14) ( } n \text { 450) }\end{array}$} & \multicolumn{2}{|c|}{$\begin{array}{c}\text { Moderate to severe } \\
\text { clinical insomnia (ISI } \\
\text { score }=15-28)(n \text { 156) }\end{array}$} & \multirow[b]{2}{*}{$P^{\star}$} \\
\hline & $n$ & $\%$ & $n$ & $\%$ & $n$ & $\%$ & $n$ & $\%$ & \\
\hline Normal (0-7) & 577 & $57 \cdot 7$ & 268 & 68.0 & 249 & $55 \cdot 3$ & 60 & 38.5 & 0.000 \\
\hline Borderline $(8-10)$ & 243 & $24 \cdot 3$ & 81 & $20 \cdot 6$ & 126 & 28.0 & 36 & $23 \cdot 1$ & \\
\hline Abnormal (11-21) & 180 & 18.0 & 45 & 11.4 & 75 & $16 \cdot 7$ & 60 & 38.5 & \\
\hline \multicolumn{10}{|c|}{ Daily dairy Ca intake (mg/d) } \\
\hline Median & $288 \cdot 12$ & & 310.08 & & $277 \cdot 28$ & & $219 \cdot 64$ & & 0.04 \\
\hline 25th-75th percentiles & $130.95-487.72$ & & $135.56-529 \cdot 76$ & & $132.79-476.48$ & & $105 \cdot 14-466 \cdot 69$ & & \\
\hline \multicolumn{10}{|c|}{ Complaint of MSP in any site of the body } \\
\hline Yes & 429 & 42.9 & 138 & 35.0 & 200 & 44.4 & 91 & $58 \cdot 3$ & 0.000 \\
\hline No & 571 & 57.1 & 256 & 65.0 & 250 & 55.6 & 65 & 41.7 & \\
\hline \multicolumn{10}{|c|}{ MSP intensity during the previous month $(0-10)$} \\
\hline Median & 0 & & 0 & & 0 & & 2 & & 0.000 \\
\hline 25th-75th percentiles & $0-4$ & & $0-2$ & & $0-4$ & & $0-\overline{5}$ & & \\
\hline \multicolumn{10}{|c|}{ Number of painful body sites (1-12) } \\
\hline Median & 0 & & 0 & & 0 & & 1 & & 0.000 \\
\hline 25th-75th percentiles & $0-1.75$ & & $0-1$ & & $0-2$ & & $0-2$ & & \\
\hline
\end{tabular}

ISI, Insomnia Severity Index; HADS, Hospital Anxiety and Depression Score; MSP, musculoskeletal pain; JD, Jordanian Dinar.

*One-way ANOVA test, Kruskal-Wallis one-way ANOVA or $\chi^{2}$ test as appropriate ( $P$-values $<0.05$ were considered statistically significant). Data are expressed as frequency (\%), mean \pm SD or median (25th-75th percentiles). †Post-hoc analysis revealed significant difference between no clinically significant insomnia and subthreshold insomnia.

severe insomnia.

$\S P o s t-h o c$ analysis revealed significant difference between subthreshold insomnia and moderate to severe insomnia.

Table 2 Correlation between ISI score and other variables ${ }^{\star}$

\begin{tabular}{|c|c|c|c|c|c|c|}
\hline & ISI score & $\begin{array}{l}\text { Daily dairy Ca intake } \\
(\mathrm{mg} / \mathrm{d})\end{array}$ & HADS-anxiety score & $\begin{array}{l}\text { HADS-depression } \\
\text { score }\end{array}$ & $\begin{array}{l}\text { MSP intensity during the } \\
\text { previous month }(0-10)\end{array}$ & $\begin{array}{l}\text { Number of painful body } \\
\text { sites }(1-12)\end{array}$ \\
\hline Age (years) & $r=0.01 ; P=0.74$ & $r=0.09 ; P<0.01$ & $r=-0.11 ; P<0.001$ & $r=-0.04 ; P=0.20$ & $r=-0.04 ; P=0.17$ & $r=-0.08 ; P=0.02$ \\
\hline BMI $\left(\mathrm{kg} / \mathrm{m}^{2}\right)$ & $r=-0.01 ; P=0.78$ & $r=0.04 ; P=0.28$ & $r=-0.02 ; P=0.65$ & $r=-0.02 ; P=0.52$ & $r=0.06 ; P=0.04$ & $r=0.03 ; P=0.44$ \\
\hline Number of family members & $r=0.05 ; P=0.12$ & $r=-0.09 ; P<0.01$ & $r=0.02 ; P=0.46$ & $r=0.07 ; P=0.02$ & $r=0.01 ; P=0.77$ & $r=-0.02 ; P=0.55$ \\
\hline ISI score & - & $r=-0.10 ; P<0.01$ & $r=0.43 ; P<0.001$ & $r=0.27 ; P<0.001$ & $r=0.24 ; P<0.001$ & $r=0.24 ; P<0.001$ \\
\hline Daily dairy $\mathrm{Ca}$ intake $(\mathrm{mg} / \mathrm{d})$ & - & - & $r=-0.06 ; P=0.06$ & $r=-0.12 ; P<0.001$ & $r=-0.01 ; P=0.73$ & $r=-0.06 ; P=0.05$ \\
\hline HADS-anxiety score & - & - & - & $r=0.52 ; P<0.001$ & $r=0.35 ; P<0.001$ & $r=0.34 ; P<0.001$ \\
\hline HADS-depression score & - & - & - & - & $r=0.24 ; P<0.001$ & $r=0.23 ; P<0.001$ \\
\hline $\begin{array}{l}\text { MSP intensity during the previous } \\
\text { month }(0-10)\end{array}$ & - & - & - & - & - & $r=0.66 ; P<0.001$ \\
\hline
\end{tabular}

month $(0-10)$ 
Table 3 Predictors of ISI score*

\begin{tabular}{|c|c|c|c|c|c|c|}
\hline Variable & $R^{2}$ & ANOVA & Model & B & $\beta$ & $P^{\star}$ \\
\hline \multirow{10}{*}{ ISI score } & \multirow{10}{*}{$0 \cdot 22$} & \multirow{10}{*}{$F=31.67, P<0.001$} & Constant & 0.72 & - & 0.62 \\
\hline & & & College & -0.03 & -0.02 & 0.52 \\
\hline & & & Smoking & 1.45 & 0.09 & 0.001 \\
\hline & & & History of medical illness during previous month & 0.03 & $<0.01$ & 0.95 \\
\hline & & & HADS-anxiety score & 0.44 & 0.35 & $<0.001$ \\
\hline & & & HADS-depression score & 0.12 & 0.08 & 0.02 \\
\hline & & & Daily dairy Ca intake & $<-0.01$ & -0.06 & 0.03 \\
\hline & & & Complaint of MSP in any site of the body & -1.22 & -0.11 & 0.04 \\
\hline & & & MSP intensity during the previous month & 0.26 & 0.12 & 0.02 \\
\hline & & & Number of painful body sites & 0.32 & $0 \cdot 10$ & 0.02 \\
\hline
\end{tabular}

ISI, Insomnia Severity Index; $R^{2}$, squared coefficient of determination; B, unstandardised coefficient; $\beta$, standardised coefficient; $F$, F-statistic; HADS, Hospital Anxiety and Depression Score; MSP, musculoskeletal pain.

*Multiple linear regression analysis $(P$-values $<0.05$ were considered statistically significant).

Table 4 Predictors of HADS-anxiety and depression scores

\begin{tabular}{|c|c|c|c|c|c|c|}
\hline Variable & $R^{2}$ & ANOVA & Model & $\mathrm{B}$ & $\beta$ & $P^{\star}$ \\
\hline \multirow[t]{10}{*}{ HADS-anxiety score } & \multirow[t]{10}{*}{0.45} & \multirow{10}{*}{$F=90 \cdot 15, P<0.001$} & Constant & -0.80 & - & 0.35 \\
\hline & & & Academic level & -0.68 & -0.08 & 0.001 \\
\hline & & & Gender & $1 \cdot 18$ & 0.13 & $<0.001$ \\
\hline & & & BMI & 0.06 & 0.06 & 0.02 \\
\hline & & & ISI score & 0.22 & 0.27 & $<0.001$ \\
\hline & & & Daily dairy $\mathrm{Ca}$ intake & $<0.001$ & 0.03 & 0.20 \\
\hline & & & HADS-depression score & 0.50 & 0.43 & $<0.001$ \\
\hline & & & Complaint of MSP in any site of the body & 0.65 & 0.07 & 0.10 \\
\hline & & & MSP intensity during the previous month & 0.11 & 0.06 & 0.17 \\
\hline & & & Number of painful body sites & $0 \cdot 18$ & 0.07 & 0.05 \\
\hline \multirow[t]{8}{*}{ HADS-depression score } & \multirow[t]{8}{*}{0.33} & \multirow[t]{8}{*}{$F=86.79, P<0.001$} & Constant & 3.54 & - & $<0.001$ \\
\hline & & & ISI score & 0.05 & 0.07 & 0.02 \\
\hline & & & Daily dairy $\mathrm{Ca}$ intake & $<-0.01$ & -0.08 & $<0.01$ \\
\hline & & & HADS-anxiety score & 0.43 & 0.50 & $<0.001$ \\
\hline & & & Complaint of MSP in any site of the body & 0.06 & 0.01 & 0.88 \\
\hline & & & MSP intensity during the previous month & 0.02 & 0.02 & 0.75 \\
\hline & & & Number of painful body sites & 0.04 & 0.02 & 0.62 \\
\hline & & & History of medical illness during last month & 0.52 & 0.04 & 0.10 \\
\hline
\end{tabular}

HADS, Hospital Anxiety and Depression Score; ISI, Insomnia Severity Index; $R^{2}$, squared coefficient of determination; B, unstandardised coefficient; $\beta$, standardised coefficient; $F, F$-statistic; MSP, musculoskeletal pain.

${ }^{*}$ Multiple linear regression analysis ( $P$-values $<0.05$ were considered statistically significant).

and HADS-depression scores $(r=0 \cdot 27, P$-value $<0.001)$ as well as between ISI and both MSP intensity during the previous month and number of painful body sites $(r=0.24$, $P$-values $<0.001)$. In contrast, daily dairy Ca intake was weakly inversely correlated with both ISI score $(r=-0 \cdot 10$, $P$-value $<0 \cdot 01)$ and HADS-depression score $(r=-0.12$, $P$-value $<0.001)$. HADS-depression score was also moderately correlated with MSP intensity during the previous month $(r=0.24, P$-value $<0.001)$ and with the number of painful body sites $(r=0.23, P$-value $<0.001)$. HADSanxiety score was also moderately correlated with MSP intensity during the previous month $(r=0 \cdot 35, P$-value $<0.001)$ and with the number of painful body sites $(r=0.34$, $P$-value $<0 \cdot 001)$

\section{Predictors of insomnia}

Multiple linear regression analysis (Table 3) indicated significant associations between ISI score and smoking $(P$-value $=0.001)$, HADS-anxiety score $(P$-value $<0.001)$,
HADS-depression score $(P$-value $=0.02)$, complaints of MSP $(P$-value $=0.04)$, MSP intensity during the previous month $(P$-value $=0.02)$ and the number of painful body sites $(P$-value $=0 \cdot 02)$. In contrast, ISI score was significantly inversely associated with daily dairy $\mathrm{Ca}$ intake $(P$-value $=0.03)$.

\section{Predictors of psychological symptoms (anxiety and depression)}

Multiple linear regression analysis (Table 4) indicated that HADS-anxiety score was significantly inversely associated with participants' academic level $(P=0.001)$ and positively associated with female gender $(P$-value $<0.001)$, BMI $(P$-value $=0.02)$, ISI score $(P$-value $<0.001)$ and HADSdepression score $(P$-value $<0 \cdot 001)$. HADS-depression score was also significantly associated with ISI score $(P$-value $=0.02)$ and HADS-anxiety score $(P$-value $<0.001)$ and was inversely associated with daily dairy Ca intake $(P$-value $<0 \cdot 01)$. 
Table 5 Predictors of MSP

\begin{tabular}{|c|c|c|c|c|c|c|}
\hline Variable & Value & $\mathrm{B}$ & SE & OR & $\mathrm{Cl}$ & $P^{*}$ \\
\hline Constant & & -3.02 & 0.48 & - & - & $<0.01$ \\
\hline Academic level & $\begin{array}{l}\text { Junior students (reference) } \\
\text { Senior students }\end{array}$ & 0.11 & $0 \cdot 14$ & $1 \cdot 12$ & $0.85,1.47$ & 0.41 \\
\hline Gender & $\begin{array}{l}\text { Male (reference) } \\
\text { Female }\end{array}$ & 0.33 & $0 \cdot 15$ & 1.39 & $1.03,1.88$ & 0.03 \\
\hline BMI & - & 0.04 & 0.02 & 1.04 & $1.01,1.07$ & 0.02 \\
\hline HADS-anxiety score & - & 0.13 & 0.02 & $1 \cdot 14$ & $1 \cdot 10,1 \cdot 19$ & $<0.001$ \\
\hline HADS-depression score & - & 0.03 & 0.02 & 1.04 & $0.99,1.08$ & 0.13 \\
\hline ISI score & - & 0.03 & 0.01 & 1.03 & $1.00,1.06$ & $<0.05$ \\
\hline
\end{tabular}

HADS, Hospital Anxiety and Depression Scale; MSP, musculoskeletal pain; ISI, Insomnia Severity Index; B, coefficient (intercept).

*Binary logistic regression (dependent variable: complaint of MSP in any site of the body), $P$-values $<0.05$ were considered statistically significant.

\section{Predictors of musculoskeletal pain}

Binary logistic regression analysis (Table 5) revealed that MSP complaints among participants were significantly associated with female gender (OR 1.39, $P$-value $=0.03)$, increased BMI (OR 1.04, $P$-value $=0.02)$, increased HADSanxiety score (OR 1.14, $P$-value $<0.001)$ and increased ISI score (OR 1.03, $P$-value $<0.05)$.

\section{Discussion}

The current study reports a high prevalence of inadequate Ca intake estimated from daily food consumption among young adults of around 21 years of age. Of concern, the median dairy $\mathrm{Ca}$ intake in our cohort of university students was $<1 / 3$ of the recommended daily intake for adults $(1000 \mathrm{mg} / \mathrm{d})^{(25)}$. Low dietary Ca intake in similar age groups has also been reported by other studies using different methods to assess dietary Ca intake ${ }^{(26,27)}$. The low dietary Ca intake among young adults could be explained by the wide spread consumption of junk food, especially in universities, that does not contain enough $\mathrm{Ca}^{(28)}$. In the present study, only dairy food contributions to Ca intake were estimated and no consideration was given to $\mathrm{Ca}$ in other foods such as green leafy vegetables or legumes with lower $\mathrm{Ca}$ content and poor bioavailability. Ca is highly available in dairy products ${ }^{(29)}$, and low dietary $\mathrm{Ca}$ intake has been linked to deleterious consequences on the skeleton leading to bone diseases such as osteoporosis ${ }^{(25)}$. In addition, low Ca intake could adversely affect other extra-skeletal functions since $\mathrm{Ca}$ is involved in muscle contraction, neurotransmission, signalling and other functions ${ }^{(30)}$. As such, our hypothesis focused on the relationship between low dairy $\mathrm{Ca}$ intake and other inter-related health problems that are commonly manifested in university students including insomnia, anxiety, depression and MSP.

Our major finding was the significant inverse association between insomnia (ISI score) and dairy Ca intake (Table 3). This association suggests that insomnia among university students can be predicted from their low intake of dairy $\mathrm{Ca}$. This finding was also supported by the significant difference in dairy $\mathrm{Ca}$ intake between participants with moderate to severe insomnia and participants with no or subthreshold insomnia (Table 1). The relationship between sleep and Ca could partially be explained by the role of brain $\mathrm{Ca}$ in converting the amino acid tryptophan to melatonin, which is considered to be a sleep-inducing hormone ${ }^{(8)}$. In addition, previous studies have indicated that $\mathrm{Ca}$ could be involved in regulating both cycles and duration of sleep in mammals ${ }^{(10)}$. Our results support the increasing evidence that suggests a role for dietary $\mathrm{Ca}$ in improving sleep quality and reducing insomnia ${ }^{(7,8)}$.

Our results have also shown that insomnia among university students can be predicted from anxiety and depression scores, MSP and MSP intensity during the previous month, number of painful body sites and smoking (Table 3). These results were supported by the significant higher anxiety and depression scores in participants with moderate to severe insomnia compared to both participants with no clinically significant insomnia and participants with subthreshold insomnia (Table 1). In addition, a higher percentage of participants who were smokers, and participants with MSP, were classified to have moderate to severe insomnia (Table 1). Moreover, the median MSP intensity and number of painful sites were significantly higher in participants with moderate to severe insomnia compared to both participants with no clinically significant insomnia and participants with subthreshold insomnia (Table 1). The relationship between insomnia and the psychological symptoms of anxiety and depression was previously reported in the literature to be bi-directional ${ }^{(31,32)}$. This was also supported by our findings as insomnia (ISI score) was a significant predictor for both anxiety and depression (Table 4) and vice versa (Table 3). This suggests that insomnia among university students may lead to anxiety and depression which in turn may cause insomnia. Insomnia and MSP were also previously reported to co-occur and each of them may increase the risk of the other ${ }^{(15)}$. Our results also supported this relationship as insomnia was a significant predictor of MSP, while MSP, MSP intensity and number of painful body sites were significant predictors of insomnia (Tables 3 and 5). The last predictor of insomnia in our study was cigarette smoking. It has been reported that sleep disturbances are more common in cigarette smokers compared 
with non-smokers ${ }^{(33)}$. This is explained by the stimulant action of nicotine that can increase alertness ${ }^{(34)}$, which may result in insomnia.

One of the main objectives of the current study was to find a link between low dietary Ca estimated from dairy food consumption and insomnia, anxiety, depression and MSP, as we believe that low Ca intake may contribute to the development of these conditions. Our results have shown that dairy $\mathrm{Ca}$ intake was inversely associated with insomnia and depression scores but not associated with anxiety scores and complaints of MSP (Tables 3, 4 and 5). These results are not completely consistent with our previous study which was conducted on subjects with non-cardiac musculoskeletal chest pain ${ }^{(14)}$. In that study, low dairy Ca intake was associated with anxiety, and anxiety was associated with pain. This inconsistency may be explained by differences in the study design and age of participants ${ }^{(14)}$. Because participants of the current study were younger and apparently healthy, their low dairy Ca intake may not result in musculoskeletal symptoms. Although there was no significant association between dairy Ca intake and anxiety or MSP, our results suggested an indirect relationship between them. The current study results indicate that anxiety may be affected by low dairy Ca intake through the significant association between anxiety and both insomnia and depression scores, which themselves are associated with low dairy Ca intake. Similarly, MSP may be affected by low dairy Ca intake through the significant association between MSP and insomnia scores, the latter of which is associated with low dairy $\mathrm{Ca}$ intake. Therefore, our results maintain support for the presence of a relationship between low dairy Ca intake, insomnia, anxiety, depression and MSP in our study population.

Taken together, the current study has exposed a relationship between low dairy $\mathrm{Ca}$ intake and medical conditions including insomnia, anxiety, depression and MSP, all of which are commonly present in university students. The main strengths of the current study were its large sample size and the established methods used to measure the variables of interest. All outcomes were determined using well-validated surveys that have been previously documented in the literature (refer to Materials and methods). The study was mainly limited by its cross-sectional design and the dependence on participants' reliable self-reporting. The study is also limited by the incomplete estimate of total dietary Ca intake, which only included dairy food sources and not that of other foods and beverages with lower $\mathrm{Ca}$ content and often poor bioavailability. Dairy foods are the most commonly consumed $\mathrm{Ca}$-rich foods by Jordanian students with highly available $\mathrm{Ca}$. These limitations may prevent us from making conclusions that can be applied to the general population. However, we believe these results are reported for the first time and hope they may encourage other researchers to further investigate the potential causal relationship between low dietary Ca intake and insomnia, anxiety, depression and MSP.
If proven true, further studies should be performed to determine if increasing dietary Ca intake, either via food choices or supplementation, is able to resolve some of these health issues among university students in order to increase both their lifestyle and scientific achievements.

\section{Conclusion}

Low dietary Ca intake estimated from dairy food consumption was highly prevalent and associated with insomnia and depression among university students from Jordan. Insomnia in our study participants could be predicted from increased anxiety and depression scores, complaint of MSP and smoking. Both anxiety and depression scores could also be predicted from insomnia scores, supporting a bi-directional association for both anxiety and depression with insomnia. Young adults should be advised to increase dietary $\mathrm{Ca}$ intake to achieve the recommended daily amount. It is also required to investigate a potential causal relationship between low dietary $\mathrm{Ca}$ and both insomnia and its related co-morbidities.

\section{Acknowledgements}

Acknowledgements: The authors would like to thank all individuals who participated in this study. Financial support: This work was financially supported by the Deanship of Research, Jordan University for Science and Technology under Grant (number: 20190367). Conflict of interest: None. Authorship: M.J.A. was responsible for study design, statistical analysis and manuscript writing. H.N.K. was responsible for data collection. K.K.A. was responsible for results interpretation and manuscript editing. Ethics of buman subject participation: This study was conducted according to the guidelines laid down in the Declaration of Helsinki, and all procedures involving research study participants were approved by the Institutional Review Board (IRB) of Jordan University of Science and Technology, Irbid, Jordan. Written informed consent was obtained from all subjects.

\section{References}

1. Buysse DJ (2013) Insomnia. JAMA 309, 706-716.

2. Saddichha S (2010) Diagnosis and treatment of chronic insomnia. Ann Indian Acad Neurol 13, 94-102.

3. Bhaskar S, Hemavathy D \& Prasad S (2016) Prevalence of chronic insomnia in adult patients and its correlation with medical comorbidities. J Family Med Prim Care 5, 780-784.

4. Javaheri S \& Redline S (2017) Insomnia and risk of cardiovascular disease. Chest 152, 435-444.

5. Mesarwi O, Polak J, Jun J et al. (2013) Sleep disorders and the development of insulin resistance and obesity. Endocrinol Metab Clin North Am 42, 617-634.

6. Rozenberg S, Body JJ, Bruyere O et al. (2016) Effects of dairy products consumption on health: benefits and beliefs-a 
commentary from the Belgian bone club and the European society for clinical and economic aspects of osteoporosis, osteoarthritis and musculoskeletal diseases. Calcif Tissue Int 98, $1-17$.

7. Grandner MA, Jackson N, Gerstner JR et al. (2014) Sleep symptoms associated with intake of specific dietary nutrients. J Sleep Res 23, 22-34.

8. Nisar M, Mohammad RM, Arshad A et al. (2019) Influence of dietary intake on sleeping patterns of medical students. Cureus 11, e4106.

9. Ridefelt P, Axelsson J \& Larsson A (2012) Diurnal variability of total calcium during normal sleep and after an acute shift of sleep. Clin Chem Lab Med 50, 147-151.

10. Tatsuki F, Sunagawa GA, Shi S et al. (2016) Involvement of $\mathrm{Ca}(2+)$-dependent hyperpolarization in sleep duration in mammals. Neuron $\mathbf{9 0}, 70-85$.

11. Kitano N, Tsunoda K, Tsuji $\mathrm{T}$ et al. (2014) Association between difficulty initiating sleep in older adults and the combination of leisure-time physical activity and consumption of milk and milk products: a cross-sectional study. BMC Geriatr 14, 118.

12. Harvey NC, Biver E, Kaufman JM et al. (2017) The role of calcium supplementation in healthy musculoskeletal ageing : An expert consensus meeting of the European Society for Clinical and Economic Aspects of Osteoporosis, Osteoarthritis and Musculoskeletal Diseases (ESCEO) and the International Foundation for Osteoporosis (IOF). Osteoporos Int 28, 447-462.

13. Schafer AL \& Shoback DM (2000) Hypocalcemia: diagnosis and treatment. [Updated 3 January 2016]. In Endotext [KR Feingold, B Anawalt, A Boyce et al., editors]. South Dartmouth, MA: MDText.com, Inc.

14. Alkhatatbeh MJ, Abdul-Razzak KK, Amara NA et al. (2019) Non-cardiac chest pain and anxiety: a possible link to vitamin D and calcium. J Clin Psychol Med Settings 26, 194-199.

15. Baker S, McBeth J, Chew-Graham CA et al. (2017) Musculoskeletal pain and co-morbid insomnia in adults; a population study of the prevalence and impact on restricted social participation. BMC Fam Pract 18, 17.

16. Khurshid KA (2018) Comorbid insomnia and psychiatric disorders: an update. Innov Clin Neurosci 15, 28-32.

17. Alvaro PK, Roberts RM \& Harris JK (2013) A systematic review assessing bidirectionality between sleep disturbances, anxiety, and depression. Sleep 36, 1059-1068.

18. Taylor DJ, Lichstein KL, Durrence HH et al. (2005) Epidemiology of insomnia, depression, and anxiety. Sleep 28, 1457-1464.

19. Oh CM, Kim HY, Na HK et al. (2019) The effect of anxiety and depression on sleep quality of individuals with high risk for insomnia: a population-based study. Front Neurol 10, 849.
20. Abdul-Razzak KK, Obeidat BA, Al-Farras MI et al. (2014) Vitamin D and PTH status among adolescent and young females with severe dysmenorrhea. I Pediatr Adolesc Gynecol 27, 78-82.

21. Bastien CH, Vallieres A \& Morin CM (2001) Validation of the Insomnia Severity Index as an outcome measure for insomnia research. Sleep Med 2, 297-307.

22. Suleiman KH \& Yates BC (2011) Translating the insomnia severity index into Arabic. J Nurs Scholarsh 43, 49-53.

23. Zigmond AS \& Snaith RP (1983) The hospital anxiety and depression scale. Acta Psychiatr Scand 67, 361-370.

24. Alkhatatbeh MJ, Abdul-Razzak KK, Khasawneh LQ et al. (2018) Prevalence of musculoskeletal pain in association with serum 25-hydroxyvitamin D concentrations in patients with type 2 diabetes mellitus. Biomed Rep 8, 571-577.

25. Cormick G \& Belizan JM (2019) Calcium intake and health. Nutrients 11, 1606.

26. Rouf AS, Sui Z, Rangan A et al. (2018) Low calcium intakes among Australian adolescents and young adults are associated with higher consumption of discretionary foods and beverages. Nutrition (Burbank, Los Angeles County, Calif) 55-56, 146-153.

27. Sandoval L, Coelho J, Jesus G et al. (2019) SUN-539 low dietary calcium intake among adolescents and young adults in Brasilia, Brazil. J Endocr Soc 3, SUN-539.

28. Lim H-S, Ji S-I, Hwang H et al. (2018) Relationship between bone density, eating habit, and nutritional intake in college students. J Bone Metab 25, 181-186.

29. Górska-Warsewicz H, Rejman K, Laskowski W et al. (2019) Milk and dairy products and their nutritional contribution to the average polish diet. Nutrients 11, 1771.

30. Agrawal A, Suryakumar G \& Rathor R (2018) Role of defective $\mathrm{Ca}(2+)$ signaling in skeletal muscle weakness: Pharmacological implications. J Cell Commun Signaling 12, 645-659.

31. Jansson-Frojmark M \& Lindblom K (2008) A bidirectional relationship between anxiety and depression, and insomnia? A prospective study in the general population. J Psychosom Res 64, 443-449.

32. Rutten S, Vriend C, van der Werf YD et al. (2017) The bidirectional longitudinal relationship between insomnia, depression and anxiety in patients with early-stage, medication-naive Parkinson's disease. Parkinsonism Relat Disord 39, 31-36.

33. Liao Y, Xie L, Chen X et al. (2019) Sleep quality in cigarette smokers and nonsmokers: findings from the general population in central China. BMC Public Health 19, 808-808.

34. Caviness CM, Anderson BJ \& Stein MD (2019) Impact of nicotine and other stimulants on sleep in young adults. $J$ Addict Med 13, 209-214. 